\title{
Mapping dysfunction of the bone marrow stroma in leukaemia
}

The bone marrow (BM)

stroma contains a variety of non-haematopoietic cells that support haematopoietic stem cell (HSC) functions during homeostasis and tissue repair. Furthermore, some BM stromal cells have been shown to directly induce myelodysplasia or leukaemia, and can be altered during leukaemia progression to promote tumour growth. Although much is known about the BM stroma and HSC niches, previous profiling studies have utilized markers or reporter genes to study only specific pre-defined cell populations.

In a study recently published in Cell, Baryawno, Przybylski, Kowalczyk et al. report single-cell RNA sequencing (scRNA-seq) analyses of the non-haematopoietic cells that comprise BM stroma. Through these analyses, they were able to more comprehensively survey the different populations of cells present in the BM stroma in mice and probe how those cells are perturbed during leukaemia development.

Unsupervised clustering of BM stroma cells from 14 C57BL/6 mice identified 17 distinct cell populations. These included cells with characteristics of mesenchymal stem cells (MSCs), two osteolineage cell (OLC) subsets and four chondrocyte subsets (OLCs and chondrocytes are derived from MSCs), five fibroblast subsets, three BM epithelial cell (BMEC) subsets and pericytes. In addition, there were

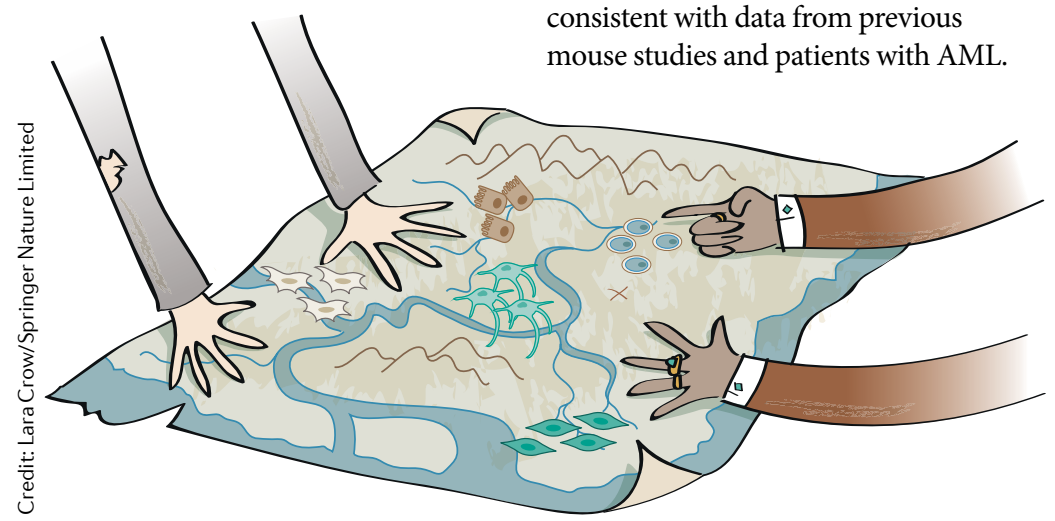

In MSCs and OLCs, expression of genes promoting differentiation was reduced, suggesting that during AML development, stromal cells as well as leukaemic cells themselves experience a differentiation block. Furthermore, genes inhibiting bone formation and calcification were induced in MSCs and OLCs, which suggests that leukaemic cells can induce bone breakdown.

The scRNA-seq profiles of BM stromal cells also indicated that normal haematopoiesis is disrupted by the presence of leukaemic cells. Several factors known to support the HSC niche, such as Cxcl12, Kitl and Angpt1, had reduced expression in some MSC, BMEC and OLC subsets. In addition, factors that are associated with decreasing HSC proliferation and pool size, such as Spp1 (which has been previously correlated with poor prognosis in patients with AML), had increased expression. This suggests that the presence of malignant cells creates a microenvironment that is not favourable for haematopoietic cells, thereby allowing leukaemic cells to outcompete them.

Interestingly, the expression of Cxcl12, Kitl and Angpt1 was increased in a subset of fibroblasts. Cancer-associated fibroblasts secreting CXCL12 have been identified in other studies to promote breast cancer metastasis; the authors propose that the fibroblast subset identified here might similarly function to promote bone metastasis, but this remains to be tested experimentally.

The stromal cell profiles identified in this study should allow further dissection of the contributions of stromal cells and crosstalk among cell types to leukaemia development and progression, and will hopefully contribute to the development of new therapeutics targeting stromal cells. They should also facilitate the use of more consistent definitions of BM stromal cells across studies.

Sarah Seton-Rogers

ORIGINAL ARTICLE Baryawno, N., Przybylski, D. Kowalczyk, M. S. et al. A cellular taxonomy of the bone marrow stroma in homeostasis and leukemia. Cell https://doi.org/10.1016/j.cell. 2019.04.040 (2019)

FURTHER READING Tikhonova, A. N., Dolgalev, I. et al. The bone marrow microenvironment at single-cell resolution. Nature 569, 222-228 (2019) 